$\mathrm{DFPD} / 02 / \mathrm{TH} / 31$

$\mathrm{ULB}-\mathrm{TH}-02 / 38$

hep-th/0212131

\title{
Massless spin-two field S-duality
}

\author{
Xavier Bekaert and Nicolas Boulanger ${ }^{\star 1}$ \\ * Dipartimento di Fisica, Università degli Studi di Padova \\ Via F. Marzolo 8, 35131 Padova, Italy \\ * Faculté des Sciences, Université Libre de Bruxelles, \\ Campus Plaine C.P. 231, B-1050 Bruxelles, Belgium
}

\begin{abstract}
We present a review of the homological algebra tools involved in the standard de Rham theory and their subsequent generalizations relevant for the understanding of free massless higher spin gauge structure. M-theory arguments suggest the existence of an extension of (Abelian) S-duality symmetry for non-Abelian gauge theories, like the four dimensional Yang-Mills or Einstein theories. Some no-go theorems prove that this extension, if it exists, should fall outside the scope of local perturbative field theory.

Proceedings of the RTN-Workshop "The quantum structure of spacetime and the geometric nature of fundamental interactions", Leuven, September 2002
\end{abstract}

\footnotetext{
1 "Chercheur F.R.I.A.", Belgium
} 


\section{$1 \quad$ Free higher spin fields}

Despite several decades of study, the problem of constructing covariant consistent interactions for higher-spin fields (i.e. spin $S>2$ ) is still only partially solved, and has turned out to be among the most intriguing and challenging problems in field theory. From the higher-spin field perspective, it is a commonplace to a posteriori view string theory as another attempt to find such a consistent interacting theory.

The birth of higher-spin field quest can be traced back to the early thirties with the pioneering work of Majorana [1] which, surprisingly enough, attracted almost no attention during three decades. In the year 1936, several authors (among whom Dirac, Klein and Proca) independently addressed the issue of field equations for particles with spin higher than one. Still, the "official" seminal paper on the subject dates from 1939 when Fierz and Pauli [2] wrote the free covariant actions for spin three-half and two. The Rarita-Schwinger spin-tensor description of spin- $\frac{3}{2}$ field followed two years later [3] (it was obtained earlier by Tamm in the thirties but he did not published his result). It was then natural to pursue the systematic study of free fields propagating in a four-dimensional Minkowski spacetime and, in the sixties, Chang 44 constructed Lagrangians for massive free fields with spin $S$ between $\frac{3}{2}$ and 4 . This work was generalized later on by Singh and Hagen for arbitrary spins [5]. In the bosonic case, this formulation requires the introduction of $S-1$ auxiliary traceless symmetric tensor fields of decreasing rank. By taking the massless limit, Fang and Fronsdal obtained in the late seventies the covariant Lagrangians for massless fields of any spin [6], elegantly described by a single gauge field subject to a double tracelessness condition. It was followed by an alternative approach to free massless higher spin fields, the so called "gauge approach" introduced by Vasilev [7, which uses geometrical objects generalizing vielbeins and spin connection. This approach turned out to be promising for switching on consistent interactions [8].

The four-dimensional case is a very special one, because all the irreps of the little group $S O(2)$ are completely symmetric. Furthermore the spin is, strictly speaking, only well-defined in four dimensions. In higher dimensions, other irreps are possible and (in the massive case) indeed appear in the spectrum of string theory. The $D \geq 4$ generalization of the previous results is direct for the two most simple irreps of the Lorentz group: (i) completely symmetric fields [9] where the $D$-dimensional massive field actions were obtained from the $(D+1)$-dimensional massless actions by standard dimensional reduction techniques [10; (ii) totally antisymmetric fields, the covariant formulation of which being easily obtained using differential forms. As we advocate in this paper, it is in fact possible to understand the gauge structure of tensor fields in an arbitrary irreps of $G L(D, \mathbb{R})$, already investigated in [1], using a systematic unified mathematical framework generalizing de Rham's theory [12, 13, 14. Note the recent work [15] based on irreps of $S L(D, \mathbb{R})$, which is then broken to its Lorentz subgroup. The surge of interest on string field theory in the mid-eighties prompted the construction of covariant action principles for free fields in exotic representations of the Lorentz group $S O(D-1,1)$ [16, 17, 18] (see [19] for a recent approach).

In a recent work on higher-spin gauge fields, Francia and Sagnotti [20] discovered that 
forgoing the orthodox locality requirement allows to relax the tracelessness conditions of the Fang-Fronsdal formulation. In this non-local approach, the higher-spin tensor gauge parameters are not constrained to be irreducible under $S O(D-1,1)$ but only under $G L(D, \mathbb{R})$. For arbitrary spin $S$, gauge invariant field equations were elegantly written [20] in terms of the curvature tensor introduced by de Wit and Freedman [9]. All known different formulations of free massless higher spin fields exhibit new features with respect to spin $S \leq 2$ fields (e.g. tracelessness conditions, auxiliary fields, non-locality, higher order kinetic operators, etc). This unavoidable novelty of higher spins is rooted in the fact that the curvature tensor contains $S$ derivatives. Therefore any local higher spin field equation built from it should be of higher order derivative. This property arises naturally when formulated in the mathematical framework of generalized complex developed by Dubois-Violette and Henneaux (for an extensive review, take a look at 21]) as was shown for completely symmetric irreps in [12, 13] and subsequently extended to arbitrary irreps in [14. We spend most part of this paper to review this mathematical machinery, that physicists are not expected to be familiar with.

\section{Algebraic background}

We start with basic definitions from homological algebra. Let $\left\{V_{i}\right\}_{i \in G}$ be a family of vector spaces indexed by an Abelian group $G$. The direct sum $V=\oplus_{i} V_{i}$ is called the $G$-graded space associated with the family $\left\{V_{i}\right\}_{i \in G}$. A differential complex is defined to be an $\mathbb{N}$-graded space $V=\bigoplus_{i \in \mathbb{N}} V_{i}$ with a nilpotent endomorphism $d$ of degree one, i.e. there is a chain of linear transformations

$$
\ldots \stackrel{d}{\longrightarrow} V_{i-1} \stackrel{d}{\longrightarrow} V_{i} \stackrel{d}{\longrightarrow} V_{i+1} \stackrel{d}{\longrightarrow} \ldots
$$

such that $d^{2}=0$. A famous example of such structure is the de Rham complex for which the vector space is the set of differential forms graded by the form degree. The role of the nilpotent operator is played by the exterior derivative $d=d x^{\mu} \partial_{\mu}$. One can now define the quotient $H(d) \equiv \frac{\operatorname{Ker}_{d}}{\operatorname{Im}_{d}}$ called the cohomology of $d$. This space inherits the grading of $V$. The elements of $H(d)$ are called (co-)cycles. Elements of $\operatorname{Im}_{d}$ are said to be trivial or exact (co)-cycles.

A straightforward generalization of the previous definitions is to consider a more complicated grading. More specifically, one takes $\mathbb{N}^{S}$ as Abelian group $(S \geq 2)$. A multicomplex is defined to be an $\mathbb{N}^{S}$-graded space $V=\oplus_{\left(i_{1}, \ldots, i_{S}\right) \in \mathbb{N}^{S}} V_{\left(i_{1}, \ldots, i_{S}\right)}$ with $S$ nilpotent endomorphisms $d_{j}(1 \leq j \leq S)$ such that

$$
d_{j} V_{\left(i_{1}, \ldots, i_{j}, \ldots, n_{S}\right)} \subset V_{\left(i_{1}, \ldots, i_{j}+1, \ldots, i_{S}\right)} .
$$

A concrete realization of this definition is the space of multiforms whose elements are sums of products of the generators $d_{j} x^{\mu}$ with smooth functions. Multiforms were recently used in free tensor gauge theories [13, 14, 22. For later purposes, we take each of the $d_{j} x^{\mu}$, $j \in\{1, \ldots, S\}$, to be commuting with all the others : $d_{i} x^{\mu} d_{j} x^{\nu}=d_{j} x^{\nu} d_{i} x^{\mu}, i \neq j$. When 
$i=j$, we demand the natural anticommutativity $d_{i} x^{\mu} d_{i} x^{\nu}=-d_{i} x^{\nu} d_{i} x^{\mu}$. The nilpotent operators $d_{j} \equiv d_{j} x^{\mu} \partial_{\mu}$ generalize the exterior differential of the de Rham complex.

An other possibility is to consider higher orders of nilpotency which allow a richer set of possibilities for cohomology. An $N$-complex is defined as a graded space $V=\oplus_{i} V_{i}$ equipped with an endomorphism $d$ of degree 1 that is nilpotent of order $N: d^{N}=0$ [21]. The generalized cohomology of the $N$-complex $V$ is the family of $N-1$ graded spaces $H_{(k)}(d)$ with $1 \leq k \leq N-1$ defined by $H_{(k)}(d)=\operatorname{Ker}\left(d^{k}\right) / \operatorname{Im}\left(d^{N-k}\right)$, i.e. $H_{(k)}(d)=$ $\oplus_{i} H_{(k)}^{i}(d)$ where

$$
H_{(k)}^{i}(d)=\left\{\phi \in V^{i} \mid d^{k} \phi=0, \phi \sim \phi+d^{N-k} \beta, \beta \in V^{p+k-N}\right\} .
$$

The multicomplex structure can be related to the $N$-complex structure in the following construction which plays a crucial role in the gauge structure of mixed symmetry type gauge fields. The idea is rather simple: to connect the two definitions one should build a $\mathbb{N}$ grading from the $\mathbb{N}^{S}$-grading of the multicomplex $\left(V, d_{j}\right)$. A simple choice is to consider the total grading defined by the sum $i \equiv \sum_{j=1}^{S} i_{j}$. We introduce the operator $d \equiv \sum_{j=1}^{S} d_{j}$ which possesses the nice property of being of total degree one. Two convenient cases arise:

- $\left[d_{i}, d_{j}\right]_{+}=0$ : Usually the nilpotent operators $d_{j}$ are taken to be anticommuting and therefore $d$ is nilpotent. This case is rather standard in homological perturbation theory.

- $\left[d_{i}, d_{j}\right]_{-}=0$ when $i \neq j$ and $d_{i}{ }^{2}=0$ : From our present perspective, commuting $d_{j}$ 's are indeed quite interesting because, in that case, $d$ is in general nilpotent of order $S+1$ and the space $V$ is endowed with a $(S+1)$-complex structure (as far as the multiforms are concerned, this convenient choice was already made in [22], while the authors of [13] chose the previous one). Indeed, every term in the expansion of $d^{S+1}$ contains at least one of the $d_{j}$ twice.

In the second case, the total cohomology group $H_{(k)}^{\left(i_{1}, \ldots, i_{S}\right)}(d)$ is the generalized cohomology group whose elements $\phi \in V_{\left(i_{1}, \ldots, i_{S}\right)}$ satisfy the set of cocycle conditions

$$
\prod_{j \in J} d_{j} \phi=0, \quad \forall J \subset\{1,2, \ldots, S\} \mid \# J=k
$$

with the equivalence relation

$$
\phi \sim \phi+\sum_{\substack{J \subset\{1,2, \ldots, S\} \\ \# J=S-k+1}} d^{J} \beta_{J},
$$

as can be seen easily by decomposing the cocycle condition $d^{k} \phi=0$ and the equivalence relation $\phi \sim \phi+d^{S-k+1} \beta$ in $\mathbb{N}^{S}$ degree. To our knowledge, the first appearance of such 
conditions was in [23] where the total cohomology was christened "hypercohomology". A sketchy definition of the total cohomology, underlying its main features, is as the quotient

$$
H_{(k)}^{\left(i_{1}, \ldots, i_{S}\right)}(d)=\frac{\bigcap \operatorname{Ker} d^{k}}{\sum \operatorname{Im} d^{S-k+1}}
$$

As a first example of generalization of Poincaré lemma, we mention that the authors of 13. proved that (it is a particular case of their theorem 2 [13] $H_{(k)}^{\left(i_{1}, \ldots, i_{S}\right)}(d)$ is trivial for $i_{j} \leq D$ in the subspace of multiforms that vanish at the origin together with all their $k$ successive derivatives.

\section{Tensor irreps of the general linear group}

Tensor irreps of $G L(D, \mathbb{R})$ are characterized by Young diagrams. A Young diagram $Y$ is a diagram which consists of a finite number $S>0$ of columns of identical squares (referred to as the cells) of finite non-increasing lengths $i_{1} \geq i_{2} \geq \ldots \geq i_{S} \geq 0$. The total number of cells of the Young diagram $Y$ is denoted by $|Y|=\sum_{j=1}^{S} i_{j}$. A Young diagram $\left(i_{1}, \ldots, i_{S}\right)$ is well-included into $\left(j_{1}, \ldots, j_{S}\right)$ iff $i_{k} \leq j_{k} \leq i_{k}+1$ for all $k \in\{1, \ldots, S\}$. In other words, the difference between two Young diagrams does not contain any column greater than a single box. We denote the well-inclusion as $\left(i_{1}, \ldots, i_{S}\right) \Subset\left(j_{1}, \ldots, j_{S}\right)$.

The set of Young diagrams with at most $S$ columns is denoted by $\mathbb{Y}^{S}$. We identify any Young diagram $Y$ with its "coordinates" $\left(i_{1}, \ldots, i_{S}\right)$. The set $\mathbb{Y}^{2}$ of Young diagrams can be pictured as the following subset of the lattice $\mathbb{N}^{2}$ :

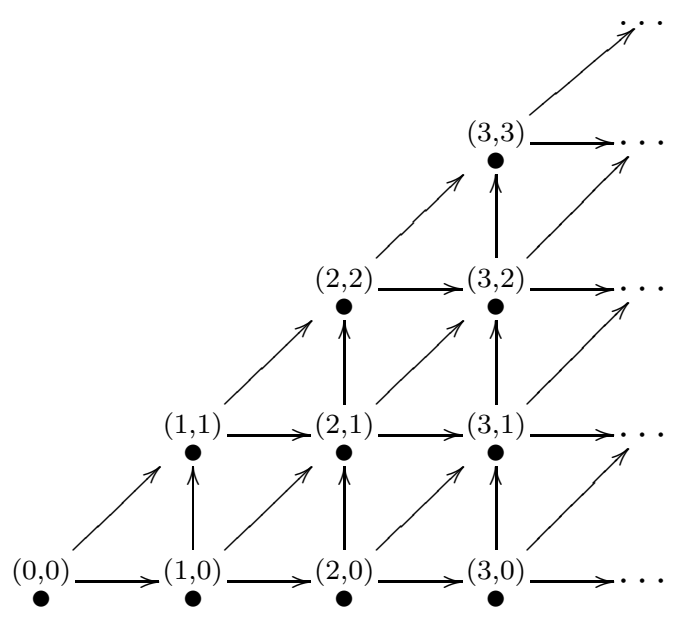

where all the arrows corresponds to well-inclusion relation $\Subset$.

To any Young diagram $Y$ is associated an operator $\mathbf{Y}$ projecting tensors of rank $|Y|$ on a definite invariant subspace $V_{Y}$ under $G L(D, \mathbb{R})$. Every cell of $Y$ is related to a specific index of the tensors in $V_{Y}$. The projector $\mathbf{Y}$ is referred to as the Young symmetrizer because the corresponding tensors possess definite symmetry properties. The Young diagrams introduce a $\mathbb{N}^{S}$-graduation of the space of tensors $V_{S}=\oplus_{Y \in \mathbb{Y} S} V_{Y}$. 
This vector space is relevant in spin $S$ theories because it contains all tensors that may be relevant in the completely symmetric tensor gauge field theory (e.g. the gauge field, its curvature, their electric-magnetic duals, the reducibility parameters, ...).

Let $Y$ and $Y^{\prime}$ be two Young diagrams in $\mathbb{Y}^{S}$ such that $Y \Subset Y^{\prime}$ and $\left|Y^{\prime}\right|=|Y|+1$. One may generalize the exterior differential by first taking the partial derivative of a tensor in $V_{Y}$ and then acting with the Young symmetrizer $\mathbf{Y}^{\prime}$. This provides a set of (at most) S commuting nilpotent maps $\mathbf{Y}^{\prime} \circ \partial$ corresponding to each available direction $Y \stackrel{\Subset}{\longrightarrow} Y^{\prime}$ in $\mathbb{Y}^{S}$. Consequently, the space $V_{S}$ is endowed with the structure of a multicomplex. Following the construction of the previous section, the total grading is the total number of cells $|Y|$ and we introduce the operator $d$ acting in each invariant subspace $V_{Y} \subset V_{S}$ as $\left.d\right|_{V_{Y}} \equiv \sum_{Y^{\prime} \ni Y} \mathbf{Y}^{\prime} \circ \partial$.

The equivalence relations (2), with $k=S$, expresses naturally the gauge freedom of an arbitrary symmetry type gauge field $\phi \in V_{S}$, associated to the invariance of the curvature tensor $R=d^{S} \phi$ expressed by (11). The higher nilpotency condition $d^{S+1}=0$ readily explains the fact that the gauge-invariant curvature $R$ contains $S$ derivatives. Therefore the gauge structure of free irreducible field theory is encoded in the cohomology group $H_{(1)}^{\left(i_{1}, \ldots, i_{S}\right)}(d)$. With this motivation in mind, we provided a theorem in [14] which proves the triviality of the generalized cohomology groups $H_{(k)}^{\left(i_{1}, \ldots, i_{S}\right)}(d)$ for $1 \leq k \leq S, 0<i_{S}$ and $i_{1} \leq D$, thereby extending the results of [12, 13].

\section{No-go theorems on non-Abelian S-duality}

Dualities are crucial in order to scrutinize non-perturbative aspects of gauge field and string theories, it is therefore of relevance to investigate the duality properties of arbitrary tensor gauge fields. It is well known that the gravity field equations in four-dimensional spacetime are formally invariant under a duality rotation. Furthermore, a deep analogy with the M5-brane toroidal compactification scenario for the self-dual D3-brane was conjectured to occur when the six-dimensional $(4,0)$ superconformal gravity theory is compactified over a 2 -torus [24]. This compactification would lead to a $S L(2, \mathbb{Z})$-duality group for four-dimensional Einstein gravity, geometrically realized as the modular group of the torus. However a naive duality rotation is not a true symmetry of gravity because the covariant derivative involves the gauge field, which is not inert under a formal duality rotation. We face the same problem in Yang-Mills theories and some no-go theorems can be established suggesting that non-Abelian S-duality may fall outside the scope of local perturbative field theory (see [25] for a short review of the spin one case).

At the free level, the electric-magnetic duality scheme works perfectly for massless tensor fields in arbitrary representations of $G L(D, \mathbb{R})$ [27, 14, 22. (duality properties of massive spin two-fields were discussed recently in [26]). The main idea is to reinterpret field equations and Bianchi identites as either

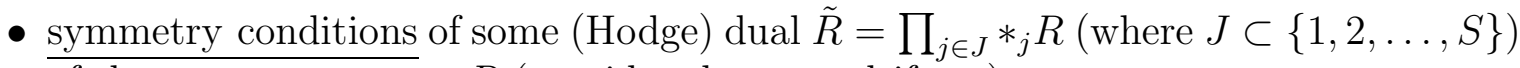
of the curvature tensor $R$ (considered as a multiform), or 
- cocycle conditions that allows to derive, from the above mentioned theorem, the existence of a dual gauge field $\tilde{\phi}$ such that $\tilde{R}=d^{S} \tilde{\phi}$.

Dualizing the Pauli-Fierz symmetric gauge field $h_{(\mu \nu)}$ in $D>4$ generates new fields in irreducible representations of $G L(D, \mathbb{R})$, characterized by mixed Young tableaux. There exists in fact two different dual fields obtained in generic spacetime dimension $D$. The first one is obtained by dualizing on one index only and involves a tensor $T_{\lambda_{1} \lambda_{2} \cdots \lambda_{D-3} \mu}$ with

$$
\begin{aligned}
& T_{\lambda_{1} \lambda_{2} \cdots \lambda_{D-3} \mu}=T_{\left[\lambda_{1} \lambda_{2} \cdots \lambda_{D-3}\right] \mu}, \\
& T_{\left[\lambda_{1} \lambda_{2} \cdots \lambda_{D-3} \mu\right]}=0
\end{aligned}
$$

where [ ] denotes antisymmetrization with strength one. The second one is obtained by dualizing on both indices and is described by a tensor $C_{\lambda_{1} \cdots \lambda_{D-3} \mu_{1} \cdots \mu_{D-3}}$ with Young symmetry type $(D-3, D-3)$ (two columns with $D-3$ boxes). Although one can write equations of motion for this theory which are equivalent to the linearized Einstein equations, these do not seem to follow (when $D>4$ ) from a Lorentz-invariant action principle in which the only varied field is $C_{\lambda_{1} \cdots \lambda_{D-3} \mu_{1} \cdots \mu_{D-3}}$. For this reason, we focus here on the dual theory based on $T_{\lambda_{1} \lambda_{2} \cdots \lambda_{D-3} \mu}$. For a recent work where massive fields possessing the aforementioned symmetries are discussed in flat and (A)dS background, see [28].

When $D=5$, the dual version of linearized gravity in terms of the field $T_{\lambda_{1} \lambda_{2} \mu}$ was given in [16], while the action for arbitrary $D$ can be found in [17]. In [29] we studied the problem of determining all the consistent, local, smooth interactions that these dual formulations in terms of $T_{\lambda_{1} \lambda_{2} \cdots \lambda_{D-3} \mu}$ gauge fields admit. It is well known that the only consistent (local, smooth) deformation of the Pauli-Fierz theory is - under quite general and reasonable assumptions - given by the Einstein theory (see the works [30, 31] for systematic analyses). Because dualization is a non-local process, one does not expect the Einstein interaction vertex to have a local counterpart on the dual $T_{\lambda_{1} \lambda_{2} \cdots \lambda_{D-3} \mu}$-side. This does not a priori preclude the existence of other local interaction vertices, which would lead to exotic self-interactions of spin-two particles. The main result of [29] is, however, that there are no Lorentz invariant deformations with at most two derivatives of the field.

\section{Acknowledgements}

X.B. thanks D. Francia and A. Sagnotti for stimulating discussions while N.B. is grateful to G. Barnich, G. Bonelli and M. Henneaux for interesting comments. The work is supported in part by the "Actions de Recherche Concertées" of the "Direction de la Recherche Scientifique - Communauté Francaise de Belgique", IISN-Belgium (convention 4.4505.86), a "Pôle d'Attraction Interuniversitaire" (Belgium) and the European Commission RTN programme HPRN-CT-2000-00131, in which N.B. is associated with K.U. Leuven. 


\section{References}

[1] E. Majorana, Nuovo Cim. 9 (1932) 335.

[2] M. Fierz and W. Pauli, Proc. Roy. Soc. Lond. A 173 (1939) 211.

[3] W. Rarita and J. S. Schwinger, Phys. Rev. 60 (1941) 61.

[4] S.-J. Chang, Phys. Rev. 161 (1967) 1308.

[5] L. P. Singh and C. R. Hagen, Phys. Rev. D 9 (1974) 898; 910.

[6] C. Fronsdal, Phys. Rev. D 18 (1978) 3624; J. Fang and C. Fronsdal, Phys. Rev. D 18 (1978) 3630;

[7] M. A. Vasiliev, Yad. Fiz. 32 (1980) 855; Fortsch. Phys. 35 (1987) 741.

[8] E. S. Fradkin and M. A. Vasiliev, Nucl. Phys. B 291 (1987) 141; Annals Phys. 177 (1987) 63.

[9] B. de Wit and D. Z. Freedman, Phys. Rev. D 21 (1980) 358.

[10] W. Siegel and B. Zwiebach, Nucl. Phys. B 263 (1986) 105; C. Aragone, S. Deser and Z. Yang, Annals Phys. 179 (1987) 76.

[11] J. M. F. Labastida and T. R. Morris, Phys. Lett. B180 (1986) 101.

[12] M. Dubois-Violette and M. Henneaux, Lett. Math. Phys. 49 (1999) 245 math.qa/9907135.

[13] M. Dubois-Violette and M. Henneaux, Commun. Math. Phys. 226 (2002) 393 math.qa/0110088.

[14] X. Bekaert and N. Boulanger, Tensor gauge fields in arbitrary representations of $G L(D, R)$ : Duality and Poincare lemma, hep-th/0208058.

[15] I. Kirsch and D. Sijacki, Class. Quant. Grav. 19 (2002) 3157, gr-qc/0111088.

[16] T. Curtright, Phys. Lett. B165 (1985) 304.

[17] C. S. Aulakh, I. G. Koh, and S. Ouvry, Phys. Lett. B173 (1986) 284.

[18] J. M. F. Labastida, Nucl. Phys. B322 (1989) 185;

[19] C. Burdik, A. Pashnev and M. Tsulaia, Mod. Phys. Lett. A 16 (2001) 731 hep-th/0101201; Nucl. Phys. Proc. Suppl. 102 (2001) 285 hep-th/0103143.

[20] D. Francia and A. Sagnotti, Phys. Lett. B 543 (2002) 303 hep-th/0207002. 
[21] M. Dubois-Violette, lectures given at the school: Bariloche 2000: Quantum Symmetries in Theoretical Physics and Mathematics, San Carlos de Bariloche, Argentina, 10-24 Jan 2000, math.qa/0005256.

[22] P. de Medeiros and C. Hull, Exotic tensor gauge theory and duality, hep-th/0208155.

[23] P.J. Olver, in S. Koh, Invariant theory, Springer-Verlag (1987) 62.

[24] C. M. Hull, Nucl. Phys. B583 (2000) 237, hep-th/0004195 Class. Quant. Grav. 18 (2001) 3233, hep-th/0011171; JHEP 12 (2000) 007, hep-th/0011215.

[25] X. Bekaert and S. Cucu, Fortsch. Phys. 50 (2002) 831 hep-th/0111215.

[26] H. Casini, R. Montemayor and L. F. Urrutia, Phys. Rev. D 66 (2002) hep-th/0206129.

[27] C. M. Hull, JHEP 09 (2001) 027 hep-th/0107149.

[28] Y. M. Zinoviev, On massive mixed symmetry tensor fields in Minkowski space and (A) $d S$, hep-th/0211233.

[29] X. Bekaert, N. Boulanger and M. Henneaux, Consistent deformations of dual formulations of linearized gravity: A no-go result, hep-th/0210278.

[30] R. M. Wald, Phys. Rev. D 33, 3613 (1986).

[31] N. Boulanger, T. Damour, L. Gualtieri, and M. Henneaux, Nucl. Phys. B597 (2001) 127, hep-th/0007220. 